\title{
EFFECT OF STEROIDS ON WATER DIURESIS AND VASOPRESSIN SENSITIVITY *
}

\author{
BY ROBERT D. LINDEMAN, $\dagger$ H. CARMER VAN BUREN $\ddagger$ AND \\ LAWRENCE G. RAISZ § \\ (From the Medical Service, Veterans Administration Hospital and Department of Medicinc, \\ State University of Nezu York Upstate Medical Center, Syracuse, N. Y.)
}

(Submitted for publication June 23, 1960 ; accepted September 15, 1960)

The ability of glucocorticoids to reverse the abnormal diuretic response to water in adrenal insufficiency (1-5) and to increase water diuresis in normal subjects $(6,7)$ is well known. Although glucocorticoids appear to be specific in this regard, the possibility that other steroids may have a pharmacologic effect on water diuresis in normal man has not been investigated systematically. The present study was therefore undertaken to compare the effects of large doses of cortisol, progesterone, estrogen and aldosterone on the maximal rate of free water clearance during water diuresis in normal man. The effects of low sodium diet and an aldosterone antagonist also were examined. It was found that only cortisol appeared to have a specific effect on this rate. Variations in maximal water diuresis also were encountered in control studies and after treatment other than cortisol, but these appeared to be related to changes in total solute excretion.

It has been suggested that glucocorticoids may antagonize the action of antidiuretic hormone (1, $6,8)$. To test this possibility, the effect of a constant infusion of a submaximal dose of exogenous vasopressin on water diuresis was examined before and after treatment with cortisol and other steroids. Variations in the response to vasopressin were encountered which were related to variations in the initial maximal rate of water diuresis in control studies. None of the agents tested, including cortisol, appeared to alter this relationship.

\footnotetext{
* Presented before the annual meeting of the American Federation for Clinical Research, Atlantic City, N. Y., May 1,1960 . Aided by grants from the National Kidney Disease Foundation and G. D. Searle \& Co.

$\dagger$ Present address: State Department of Health, Oklahoma City, Okla.

$\$$ Present address : 19 Woodlawn Road, Burlington, Vt.

$\S$ Veterans Administration Clinical Investigator. Present address: Strangeways Research Laboratory, Cambridge, England.
}

\section{METHODS}

Five healthy, paid volunteer medical students served as subjects. The experiments were performed in the postabsorptive state, but at different times of day. After an initial oral water load of $20 \mathrm{ml}$ per $\mathrm{kg}$ of body weight, the subject maintained water diuresis by drinking an amount of water equal to the amount voided during the last urine collection period. Urine collections were obtained by voluntary voiding at 15 -minute intervals. An infusion of 5 per cent dextrose in water was maintained at a constant rate of 0.6 or $1.2 \mathrm{ml}$ per minute throughout the experiment. Blood samples for determination of total solute, electrolyte and creatinine concentration were obtained at the beginning and the end of the infusion. In order to minimize antidiuretic stimuli, no injection or venipuncture was performed during the experiment except for 3 experiments in Subject L.B., in which inulin clearance was measured. After a steady rate of water diuresis had been established for at least 3 periods, the infusion was changed to one containing vasopressin in an amount to provide $4,8,12$ or $16 \mathrm{mU}$ per hour. A freshly opened ampule of commercial vasopressin (Pitressin; Parke, Davis \& Co.) was used for each experiment. One lot (S216F) was used for 4 subjects. The lot used in Subject L.B. (Y108HA) had less antidiuretic potency when the two lots were compared in the same experiment. In most experiments, the response to a single rate of vasopressin administration was observed. In selected experiments, two different rates were used. Each rate was continued for 90 to 120 minutes, until the urine flow was again steady. After vasopressin was discontinued, the return to a new steady state of maximal water diuresis was observed. This usually required 75 to 90 minutes. The data from such experiments are presented as the mean values for 2 to 4 consecutive periods in which urine flow did not vary by more than 10 per cent during maximal water diuresis, or by more than $0.5 \mathrm{ml}$ per minute during vasopressin infusion.

In addition to 3 or 4 control experiments in each subject, the experiments were repeated after the following forms of treatment.

1. Cortisol. Three subjects took $40 \mathrm{mg}$ of cortisol orally every 6 hours for 4 days.

2. Progesterone. Two subjects received intramuscular injections of $100 \mathrm{mg}$ of progesterone in oil every 12 hours for 4 days.

3. Estrogen. Two subjects took $0.1 \mathrm{mg}$ of ethinyl estradiol orally every 6 hours for 7 days. 
4. Aldosterone. Three subjects received intramuscular injections of $0.3 \mathrm{mg}$ of $d l$-aldosterone monoacetate every 6 hours for 3 days. A fourth subject (R.B.) was studied after 1 day of aldosterone administration.

5. Low salt diet. Three subjects ingested a $200 \mathrm{mg}$ sodium diet for 3 to 5 days prior to study.

6. Spironolactone. At the completion of the experiment on a low sodium diet, these subjects took $200 \mathrm{mg}$ of spironolactone, an aldosterone antagonist, orally every 6 hours for 2 days while remaining on a low sodium intake. A fourth subject (A.G.) was studied after receiving spironolactone for 4 days while on an unrestricted diet.

\section{Chemical methods and calculations}

Methods for determination of osmolality, creatinine, sodium and potassium have been described previously from this laboratory (9). The net excess of water in the urine, or free water clearance $\left(\mathrm{C}_{\mathrm{H}_{2} \mathrm{O}}\right)$, was calculated as the difference between urine flow and osmolal clearance. Creatinine clearance values served as a check on the constancy of bladder emptying and as a rough measure of glomerular filtration rate. Inulin clearance (10) was measured in three experiments in Subject L.B. Antidiuresis occurred with blood sampling and at the time of injection of the inulin prime, and the experiments had to be prolonged in order to obtain data on steady state water diuresis. For this reason, inulin clearance was not measured in any other experiments. The response to vasopressin was measured as the change in free water clearance which occurred between the ini- tial maximal water diuresis and the steady state during vasopressin infusion. This has been termed $\Delta \mathrm{C}_{\mathrm{H}_{2} \mathrm{O}}$ (11).

\section{RESULTS}

The results are summarized in Table I.

I. Maximal water diuresis. Values for free water clearance during maximal water diuresis varied considerably. In control studies, changes in maximal $\mathrm{C}_{\mathrm{H}_{2} \mathrm{O}}$ could be related to variations in total solute excretion (Figure 1). This relationship was observed whether the variation in solute excretion could be related to diurnal rhythm or to a change in dietary intake. Although sodium excretion and total solute excretion often were parallel, the former showed a greater variability, and the correlation between sodium excretion and $\mathrm{C}_{\mathrm{H}_{2} \mathrm{O}}$ was not significant in control experiments. Data obtained after progesterone and estrogen administration showed slightly high values for sodium excretion, total solute excretion and $\mathrm{C}_{\mathrm{H}_{2} \mathrm{O}}$. After intramuscular administration of aldosterone, sodium excretion was somewhat decreased compared with the mean control values, and potassium excretion was increased. However, total solute excretion and $\mathrm{C}_{\mathrm{H}_{2} \mathrm{O}}$ decreased only in Subject L.B. A marked decrease in sodium excretion and a de-

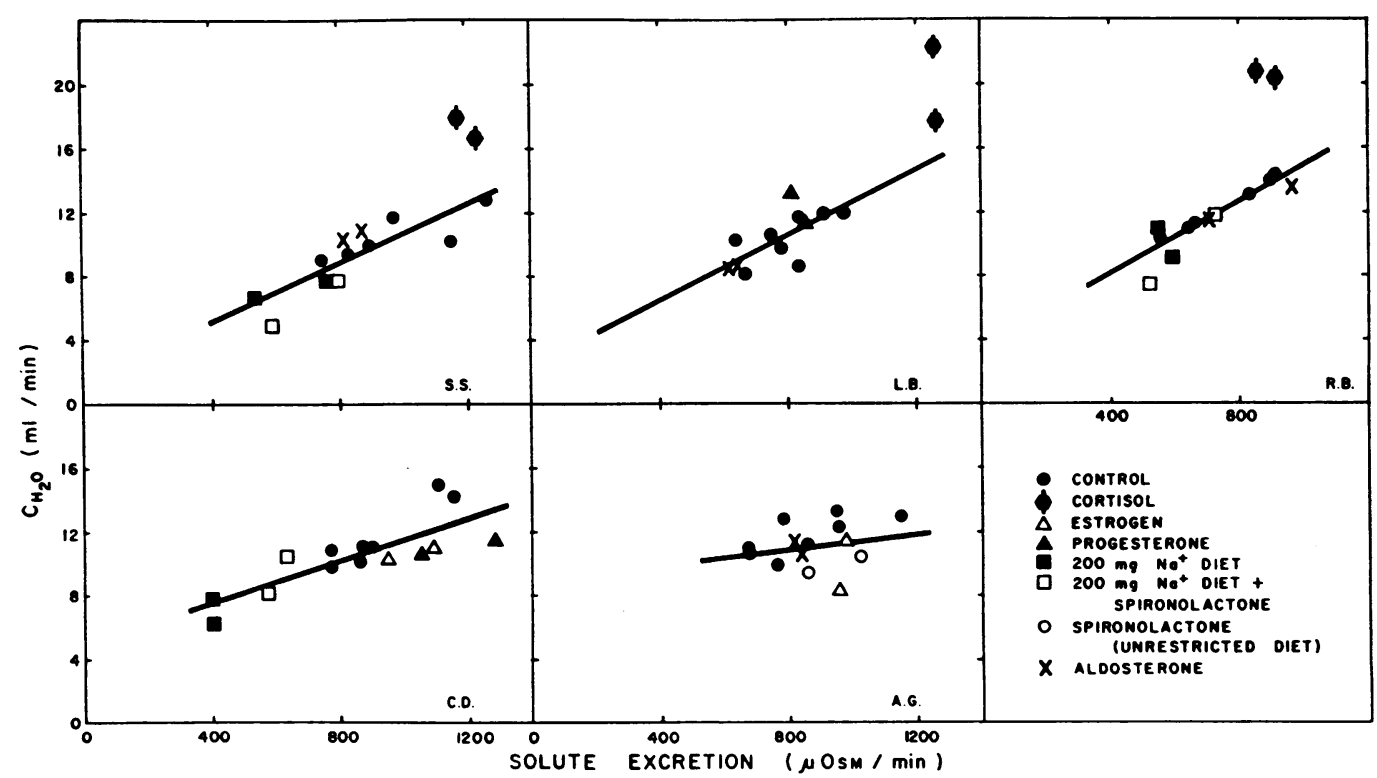

Fig. 1. Maximal free water clearance $\left(\mathrm{C}_{\mathrm{H}: \mathrm{O}}\right)$ versus total solute excretion. Data are shown for the subjects individually before and after the administration of cortisol, estrogen, progesterone, aldosterone, a low sodium diet and an aldosterone antagonist. The solid lines represent the regression line calculated by the method of least squares for all data for each subject exclusive of values obtained after cortisol administration. 
TABLE I

Water diuresis and vasopressin response in normal young men. Control studies and effect of cortisol (Cort), progesterone (Prog), estrogen (Estr), aldosterone (Ald), $200 \mathrm{mg}$ sodium diet (Low Na) and spironolactone on low sodium diet $(S p+$ low $N a)$ or on unrestricted diet $(S p)$ are shown. Time given is for the beginning of vasopressin infusion

\begin{tabular}{|c|c|c|c|c|c|c|c|c|c|}
\hline $\begin{array}{l}\text { Subject } \\
\text { Age } \\
\text { Surface Area }\end{array}$ & $\begin{array}{l}\text { Study } \\
\text { Date } \\
\text { Time } \\
\end{array}$ & $\begin{array}{l}\text { Vaso- } \\
\text { pressin } \\
\mathrm{mu} / \mathrm{hr} \\
\end{array}$ & $\begin{array}{l}\text { Urine } \\
\text { Flow } \\
\mathrm{ml} / \mathrm{min}\end{array}$ & $\begin{array}{l}\text { Urine } \\
\text { Osmolality } \\
\mathrm{mOsm} / \mathrm{L} \\
\end{array}$ & $\begin{array}{l}\text { Solute } \\
\text { Excretion } \\
\text { Hosm/min }\end{array}$ & $\begin{array}{l}\mathrm{CH}_{2} \mathrm{O} \\
\mathrm{ml} / \mathrm{min}\end{array}$ & $\begin{array}{l}\text { Sodium } \\
\text { Excretion } \\
\mu E q / \min \\
\end{array}$ & $\begin{array}{l}\text { Creatinine } \\
\text { Clearance } \\
\mathrm{ml} / \mathrm{min} \\
\end{array}$ & $\begin{array}{l}\triangle \mathrm{CH}_{2} \mathrm{O} \\
\mathrm{ml} / \mathrm{min}\end{array}$ \\
\hline $\begin{array}{l}\text { S. S. } \\
24 \\
1.98 \mathrm{~m}^{2}\end{array}$ & $\begin{array}{l}\text { Control } \\
10 / 16 / 59 \\
9: 30 \mathrm{am}\end{array}$ & $\begin{array}{l}0 \\
4 \\
0\end{array}$ & $\begin{array}{r}15.0 \\
6.8 \\
13.1\end{array}$ & $\begin{array}{r}65 \\
158 \\
68\end{array}$ & $\begin{array}{r}974 \\
1071 \\
896\end{array}$ & $\begin{array}{r}11.6 \\
3.0 \\
9.9\end{array}$ & $\begin{array}{l}126 \\
194 \\
130\end{array}$ & $\begin{array}{r}104 \\
99 \\
100\end{array}$ & 8.6 \\
\hline & $\begin{array}{l}\text { Cort } \\
10 / 23 / 59 \\
9: 45 \mathrm{am}\end{array}$ & $\begin{array}{l}0 \\
4 \\
8 \\
0\end{array}$ & $\begin{array}{r}20.5 \\
8.5 \\
2.8 \\
21.8\end{array}$ & $\begin{array}{r}60 \\
98 \\
312 \\
53\end{array}$ & $\begin{array}{r}1230 \\
837 \\
874 \\
1155\end{array}$ & $\begin{array}{r}16.3 \\
5.6 \\
-.3 \\
17.7\end{array}$ & $\begin{array}{r}240 \\
71 \\
120 \\
292\end{array}$ & $\begin{array}{l}146 \\
120 \\
140 \\
155\end{array}$ & $\begin{array}{l}10.7 \\
16.6\end{array}$ \\
\hline & $\begin{array}{l}\text { Control } \\
11 / 2 / 59 \\
9: 45 \mathrm{am}\end{array}$ & $\begin{array}{l}0 \\
4 \\
8 \\
0\end{array}$ & $\begin{array}{r}16.8 \\
4.5 \\
1.7 \\
14.0\end{array}$ & $\begin{array}{r}76 \\
190 \\
469 \\
78\end{array}$ & $\begin{array}{r}1265 \\
849 \\
769 \\
1102\end{array}$ & $\begin{array}{r}12.3 \\
1.5 \\
-1.1 \\
10.1\end{array}$ & $\begin{array}{l}189 \\
190 \\
234 \\
305\end{array}$ & $\begin{array}{l}123 \\
121 \\
119 \\
112\end{array}$ & $\begin{array}{l}10.8 \\
13.4\end{array}$ \\
\hline & $\begin{array}{l}\frac{\text { Low } \mathrm{Na}}{11 / 6 / 59} \\
10: 15 \mathrm{am}\end{array}$ & $\begin{array}{l}0 \\
4 \\
0\end{array}$ & $\begin{array}{r}10.4 \\
3.1 \\
8.5\end{array}$ & $\begin{array}{r}73 \\
167 \\
64\end{array}$ & $\begin{array}{l}760 \\
512 \\
538\end{array}$ & $\begin{array}{l}7.7 \\
1.2 \\
6.5\end{array}$ & $\begin{array}{r}7 \\
12 \\
41\end{array}$ & $\begin{array}{l}115 \\
104 \\
107\end{array}$ & 6.5 \\
\hline & $\begin{array}{l}\frac{S p+l o w ~ N a}{11 / 9 / 59} \\
10: 30 \mathrm{am}\end{array}$ & $\begin{array}{l}0 \\
4 \\
0\end{array}$ & $\begin{array}{r}10.5 \\
2.4 \\
6.8\end{array}$ & $\begin{array}{r}76 \\
254 \\
87\end{array}$ & $\begin{array}{l}798 \\
613 \\
596\end{array}$ & $\begin{array}{l}7.7 \\
.2 \\
4.7\end{array}$ & $\begin{array}{r}110 \\
78 \\
70\end{array}$ & $\begin{array}{l}112 \\
103\end{array}$ & 7.5 \\
\hline & $\begin{array}{l}\frac{\text { Control }}{2 / 27 / 60} \\
12: 45 \mathrm{pm}\end{array}$ & $\begin{array}{l}0 \\
4 \\
0\end{array}$ & $\begin{array}{r}11.6 \\
4.1 \\
12.3\end{array}$ & $\begin{array}{r}64 \\
164 \\
68\end{array}$ & $\begin{array}{l}748 \\
675 \\
828\end{array}$ & $\begin{array}{l}8.9 \\
1.7 \\
9.3\end{array}$ & $\begin{array}{r}43 \\
128 \\
146\end{array}$ & $\begin{array}{l}104 \\
117 \\
119\end{array}$ & 7.2 \\
\hline & $\begin{array}{l}\text { Ald } \\
3 / 5 / 59 \\
1: 30 \mathrm{pm}\end{array}$ & $\begin{array}{l}0 \\
4 \\
0\end{array}$ & $\begin{array}{c}14.1 \\
4.9 \\
13.9\end{array}$ & $\begin{array}{r}58 \\
151 \\
62\end{array}$ & $\begin{array}{l}817 \\
744 \\
870\end{array}$ & $\begin{array}{r}11.2 \\
2.3 \\
10.8\end{array}$ & $\begin{array}{r}76 \\
84 \\
158\end{array}$ & $\begin{array}{l}122 \\
113 \\
119\end{array}$ & 8.9 \\
\hline $\begin{array}{l}\text { C. D. } \\
24 \\
2.03 \mathrm{~m}^{2}\end{array}$ & $\begin{array}{l}\text { Control } \\
7 / 22 / 59 \\
9: 45 \mathrm{am}\end{array}$ & $\begin{array}{l}0 \\
8 \\
0\end{array}$ & $\begin{array}{r}18.8 \\
5.4 \\
12.6\end{array}$ & $\begin{array}{r}58 \\
168 \\
61\end{array}$ & $\begin{array}{r}1100 \\
895 \\
769\end{array}$ & $\begin{array}{r}14.9 \\
2.1 \\
9.8\end{array}$ & $\begin{array}{l}120 \\
115 \\
160\end{array}$ & $\begin{array}{l}155 \\
142 \\
191\end{array}$ & 12.8 \\
\hline & $\begin{array}{l}\frac{\text { Prog }}{7 / 29 / 59} \\
10: 00 \mathrm{am}\end{array}$ & $\begin{array}{l}0 \\
8 \\
0\end{array}$ & $\begin{array}{r}16.0 \\
4.4 \\
14.3\end{array}$ & $\begin{array}{r}79 \\
238 \\
73\end{array}$ & $\begin{array}{l}1280 \\
1060 \\
1055\end{array}$ & $\begin{array}{r}11.5 \\
0.6 \\
10.5\end{array}$ & $\begin{array}{l}201 \\
230 \\
175\end{array}$ & $\begin{array}{l}144 \\
128 \\
127\end{array}$ & 10.9 \\
\hline & $\begin{array}{l}\frac{\text { Control }}{10 / 2 / 59} \\
2: 30 \mathrm{pm}\end{array}$ & $\begin{array}{l}0 \\
8 \\
0\end{array}$ & $\begin{array}{r}14.2 \\
5.0 \\
14.3\end{array}$ & $\begin{array}{r}63 \\
172 \\
62\end{array}$ & $\begin{array}{l}896 \\
860 \\
870\end{array}$ & $\begin{array}{r}11.0 \\
11.9 \\
11.1\end{array}$ & $\begin{array}{r}72 \\
152 \\
137\end{array}$ & $\begin{array}{l}122 \\
144 \\
138\end{array}$ & 9.1 \\
\hline & $\begin{array}{l}\text { Estr } \\
10 / 9 / 59 \\
1: 30 \mathrm{pm}\end{array}$ & $\begin{array}{l}0 \\
8 \\
0\end{array}$ & $\begin{array}{r}14.8 \\
5.5 \\
13.7\end{array}$ & $\begin{array}{r}73 \\
205 \\
69\end{array}$ & $\begin{array}{r}1083 \\
1121 \\
944\end{array}$ & $\begin{array}{r}10.9 \\
1.4 \\
10.3\end{array}$ & $\begin{array}{l}188 \\
282 \\
202\end{array}$ & $\begin{array}{l}138 \\
161 \\
145\end{array}$ & 9.5 \\
\hline & $\begin{array}{l}\text { Control } \\
\frac{11 / 18 / 59}{10: 30 \mathrm{am}}\end{array}$ & $\begin{array}{l}0 \\
8 \\
0\end{array}$ & $\begin{array}{r}18.3 \\
5.2 \\
13.6\end{array}$ & $\begin{array}{c}63 \\
150 \\
54\end{array}$ & $\begin{array}{r}1147 \\
772 \\
738\end{array}$ & $\begin{array}{r}14.1 \\
2.3 \\
10.8\end{array}$ & $\begin{array}{r}161 \\
99 \\
106\end{array}$ & $\begin{array}{l}102 \\
112 \\
123\end{array}$ & 11.8 \\
\hline & $\begin{array}{l}\frac{\text { Low Na }}{11 / 23 / 59} \\
1: 30 \mathrm{pm}\end{array}$ & $\begin{array}{l}0 \\
8 \\
0\end{array}$ & $\begin{array}{l}7.8 \\
0.7 \\
9.3\end{array}$ & $\begin{array}{r}52 \\
479 \\
44\end{array}$ & $\begin{array}{l}404 \\
356 \\
404\end{array}$ & $\begin{array}{l}6.3 \\
-.6 \\
7.8\end{array}$ & $\begin{array}{r}9 \\
2 \\
12\end{array}$ & $\begin{array}{l}148 \\
152 \\
126\end{array}$ & 6.9 \\
\hline & $\begin{array}{l}\frac{\text { Sp+low Na }}{11 / 25 / 59} \\
10: 00 \mathrm{am}\end{array}$ & $\begin{array}{l}0 \\
8 \\
0\end{array}$ & $\begin{array}{r}12.8 \\
3.4 \\
10.3\end{array}$ & $\begin{array}{r}50 \\
193 \\
56\end{array}$ & $\begin{array}{l}635 \\
652 \\
578\end{array}$ & $\begin{array}{r}10.4 \\
0.9 \\
8.1\end{array}$ & $\begin{array}{l}41 \\
65 \\
40\end{array}$ & $\begin{array}{r}128 \\
132 \\
87\end{array}$ & 9.5 \\
\hline $\begin{array}{l}\text { R. B. } \\
24 \\
1.80 \mathrm{~m}^{2}\end{array}$ & $\begin{array}{l}\text { Control } \\
11 / 4 / 59 \\
9: 45 \mathrm{am}\end{array}$ & $\begin{array}{l}0 \\
4 \\
8 \\
0\end{array}$ & $\begin{array}{r}13.6 \\
8.6 \\
2.0 \\
17.2\end{array}$ & $\begin{array}{r}49 \\
90 \\
348 \\
52\end{array}$ & $\begin{array}{l}665 \\
774 \\
696 \\
895\end{array}$ & $\begin{array}{r}11.2 \\
5.8 \\
-.5 \\
14.0\end{array}$ & $\begin{array}{c}43 \\
97 \\
124 \\
172\end{array}$ & $\begin{array}{r}89 \\
101 \\
99 \\
102\end{array}$ & $\begin{array}{r}5.4 \\
11.7\end{array}$ \\
\hline & $\begin{array}{l}\text { Cort } \\
11 / 14 / 59 \\
12: 45 \mathrm{pm}\end{array}$ & $\begin{array}{l}0 \\
4 \\
8 \\
0\end{array}$ & $\begin{array}{r}23.5 \\
14.6 \\
6.6 \\
23.8\end{array}$ & $\begin{array}{r}39 \\
56 \\
121 \\
36\end{array}$ & $\begin{array}{l}916 \\
817 \\
802 \\
856\end{array}$ & $\begin{array}{r}20.2 \\
11.6 \\
3.6 \\
20.6\end{array}$ & $\begin{array}{r}91 \\
92 \\
112 \\
169\end{array}$ & $\begin{array}{r}105 \\
101 \\
78 \\
110\end{array}$ & $\begin{array}{r}8.6 \\
16.6\end{array}$ \\
\hline & $\begin{array}{l}\text { Control } \\
11 / 27 / 59 \\
11: 15 \mathrm{am}\end{array}$ & $\begin{array}{l}0 \\
4 \\
0\end{array}$ & $\begin{array}{r}12.4 \\
7.4 \\
15.6\end{array}$ & $\begin{array}{l}45 \\
92 \\
42\end{array}$ & $\begin{array}{l}558 \\
677 \\
646\end{array}$ & $\begin{array}{r}10.3 \\
4.8 \\
13.0\end{array}$ & $\begin{array}{r}25 \\
101 \\
70\end{array}$ & $\begin{array}{l}78 \\
83\end{array}$ & 5.5 \\
\hline & $\begin{array}{l}\frac{\text { Low } \mathrm{Na}}{11 / 30 / 59} \\
1: 30 \mathrm{pm}\end{array}$ & $\begin{array}{l}0 \\
4 \\
0\end{array}$ & $\begin{array}{r}11.3 \\
4.7 \\
13.0\end{array}$ & $\begin{array}{r}54 \\
122 \\
42\end{array}$ & $\begin{array}{l}597 \\
572 \\
546\end{array}$ & $\begin{array}{r}9.1 \\
2.6 \\
10.9\end{array}$ & $\begin{array}{l}4 \\
7 \\
8\end{array}$ & $\begin{array}{l}97 \\
95\end{array}$ & 6.5 \\
\hline
\end{tabular}


Table I. (cont.)

\begin{tabular}{|c|c|c|c|c|c|c|c|c|c|}
\hline $\begin{array}{l}\text { Subject } \\
\text { Age } \\
\text { Surface Area } \\
\end{array}$ & $\begin{array}{l}\text { Study } \\
\text { Date } \\
\text { Time } \\
\end{array}$ & $\begin{array}{l}\text { Vaso- } \\
\text { pressin } \\
\mathrm{mu} / \mathrm{hr} \\
\end{array}$ & $\begin{array}{l}\text { Urine } \\
\text { Flow } \\
\mathrm{ml} / \mathrm{min} \\
\end{array}$ & $\begin{array}{l}\text { Urine } \\
\text { Osmolality } \\
\mathrm{mOsm} / \mathrm{L} \\
\end{array}$ & $\begin{array}{l}\text { Solute } \\
\text { Excretion } \\
\text { LOsm/min }\end{array}$ & $\begin{array}{l}\mathrm{CH}_{2} \mathrm{O} \\
\mathrm{ml} / \mathrm{min} \\
\end{array}$ & $\begin{array}{l}\text { Sodium } \\
\text { Excretion } \\
\mu E_{q} / \min \\
\end{array}$ & $\begin{array}{l}\text { Creatinine } \\
\text { Clearance } \\
\mathrm{ml} / \mathrm{min} \\
\end{array}$ & $\begin{array}{l}\mathrm{OCH}_{2} \mathrm{O} \\
\mathrm{ml} / \mathrm{min}\end{array}$ \\
\hline \multirow[t]{3}{*}{ R. B. (cont.) } & $\begin{array}{l}\frac{S p+i o w ~ N a}{12 / 2 / 59} \\
10: 30 \mathrm{am}\end{array}$ & $\begin{array}{l}0 \\
4 \\
0\end{array}$ & $\begin{array}{r}9.2 \\
4.5 \\
14.2\end{array}$ & $\begin{array}{r}57 \\
117 \\
50\end{array}$ & $\begin{array}{l}525 \\
531 \\
704\end{array}$ & $\begin{array}{r}7.3 \\
2.5 \\
11.6\end{array}$ & $\begin{array}{l}19 \\
35 \\
83\end{array}$ & $\begin{array}{l}99 \\
94 \\
98\end{array}$ & 4.8 \\
\hline & $\begin{array}{l}\text { Control } \\
\frac{12 / 19 / 59}{10: 30 \mathrm{am}}\end{array}$ & $\begin{array}{l}0 \\
4 \\
0\end{array}$ & $\begin{array}{l}15.9 \\
10.2 \\
17.4\end{array}$ & $\begin{array}{l}52 \\
92 \\
52\end{array}$ & $\begin{array}{l}832 \\
950 \\
907\end{array}$ & $\begin{array}{r}12.9 \\
6.8 \\
14.1\end{array}$ & $\begin{array}{r}77 \\
196 \\
305\end{array}$ & $\begin{array}{l}105 \\
100 \\
110\end{array}$ & 6.1 \\
\hline & $\begin{array}{l}\text { Ald } \\
12728 / 59 \\
10: 30 \mathrm{am}\end{array}$ & $\begin{array}{l}0 \\
4 \\
0\end{array}$ & $\begin{array}{r}14.0 \\
8.7 \\
16.9\end{array}$ & $\begin{array}{r}50 \\
105 \\
57\end{array}$ & $\begin{array}{l}706 \\
911 \\
965\end{array}$ & $\begin{array}{r}11.5 \\
5.4 \\
13.4\end{array}$ & $\begin{array}{r}38 \\
116 \\
155\end{array}$ & $\begin{array}{l}103 \\
105 \\
102\end{array}$ & 6.1 \\
\hline \multirow[t]{7}{*}{$\begin{array}{l}\text { A.G. } \\
25 \\
1.80 \mathrm{~m}^{2}\end{array}$} & $\begin{array}{l}\text { Control } \\
4 / 17 / 59 \\
10: 45 \mathrm{am}\end{array}$ & $\begin{array}{l}0 \\
8 \\
c\end{array}$ & $\begin{array}{r}16.9 \\
3.3 \\
15.6\end{array}$ & $\begin{array}{r}68 \\
240 \\
50\end{array}$ & $\begin{array}{r}1150 \\
800 \\
\therefore \quad 782\end{array}$ & $\begin{array}{r}12.9 \\
.5 \\
12.8\end{array}$ & $\begin{array}{l}286 \\
162 \\
142\end{array}$ & $\begin{array}{l}146 \\
127 \\
172\end{array}$ & 12.4 \\
\hline & $\begin{array}{l}\frac{\text { Control }}{9 / 11 / 59} \\
10: 00 \mathrm{am}\end{array}$ & $\begin{array}{l}0 \\
8 \\
0\end{array}$ & $\begin{array}{r}15.6 \\
2.5 \\
14.2\end{array}$ & $\begin{array}{r}61 \\
286 \\
61\end{array}$ & $\begin{array}{l}959 \\
720 \\
862\end{array}$ & $\begin{array}{r}12.2 \\
-\ddot{1} 1 \\
11.1\end{array}$ & $\begin{array}{r}95 \\
140 \\
164\end{array}$ & $\begin{array}{l}144 \\
149 \\
172\end{array}$ & 12.3 \\
\hline & $\begin{array}{l}\frac{\text { Estr }}{9 / 20 / 59} \\
10: 45 \mathrm{am}\end{array}$ & $\begin{array}{l}0 \\
8 \\
0\end{array}$ & $\begin{array}{r}14.7 \\
4.1 \\
11.7\end{array}$ & $\begin{array}{r}66 \\
290 \\
82\end{array}$ & $\begin{array}{r}978 \\
1178 \\
950\end{array}$ & $\begin{array}{r}11.3 \\
-.2 \\
8.2\end{array}$ & $\begin{array}{l}126 \\
210 \\
178\end{array}$ & $\begin{array}{l}208 \\
181 \\
142\end{array}$ & 11.5 \\
\hline & $\begin{array}{l}\text { Control } \\
10 / 5 / 59 \\
10: 45 \mathrm{am}\end{array}$ & $\begin{array}{l}0 \\
8 \\
0\end{array}$ & $\begin{array}{r}13.2 \\
2.4 \\
16.7\end{array}$ & $\begin{array}{r}51 \\
268 \\
56\end{array}$ & $\begin{array}{l}675 \\
623 \\
951\end{array}$ & $\begin{array}{r}10.8 \\
.1 \\
13.2\end{array}$ & $\begin{array}{r}72 \\
116 \\
225\end{array}$ & $\begin{array}{l}171 \\
150 \\
179\end{array}$ & 10.7 \\
\hline & $\begin{array}{l}\frac{S p}{10 / 10 / 59} \\
11: 00 \mathrm{am}\end{array}$ & $\begin{array}{l}0 \\
8 \\
0\end{array}$ & $\begin{array}{r}14.1 \\
3.0 \\
12.4\end{array}$ & $\begin{array}{r}72 \\
316 \\
70\end{array}$ & $\begin{array}{r}1021 \\
947 \\
860\end{array}$ & $\begin{array}{r}10.4 \\
-.4 \\
9.3\end{array}$ & $\begin{array}{l}184 \\
200 \\
155\end{array}$ & $\begin{array}{l}148 \\
142 \\
154\end{array}$ & 10.8 \\
\hline & $\begin{array}{l}\text { Control } \\
\text { 11/16/59 } \\
\text { 2:00 pm }\end{array}$ & $\begin{array}{l}0 \\
8 \\
0\end{array}$ & $\begin{array}{r}12.5 \\
1.1 \\
13.2\end{array}$ & $\begin{array}{r}62 \\
551 \\
51\end{array}$ & $\begin{array}{l}765 \\
584 \\
673\end{array}$ & $\begin{array}{r}9.8 \\
-1.1 \\
10.7\end{array}$ & $\begin{array}{r}68 \\
55 \\
140\end{array}$ & & 10.9 \\
\hline & $\begin{array}{l}\frac{\text { Ald }}{3 / 5 / 60} \\
10: 30 \text { am }\end{array}$ & $\begin{array}{l}0 \\
8 \\
0\end{array}$ & $\begin{array}{r}13.8 \\
2.8 \\
13.6\end{array}$ & $\begin{array}{r}60 \\
300 \\
62\end{array}$ & $\begin{array}{l}818 \\
830 \\
842\end{array}$ & $\begin{array}{r}10.8 \\
-.2 \\
10.6\end{array}$ & $\begin{array}{l}103 \\
106 \\
103\end{array}$ & & 11.0 \\
\hline \multirow[t]{7}{*}{$\begin{array}{l}\text { L. B. } \\
26 \\
2.00 \mathrm{~m}^{2}\end{array}$} & $\begin{array}{l}\text { Control } \\
12 / 31 / 59 \\
1: 45 \mathrm{pm}\end{array}$ & $\begin{array}{l}0 \\
8\end{array}$ & $\begin{array}{l}14.7 \\
12.4\end{array}$ & $\begin{array}{l}56 \\
67\end{array}$ & $\begin{array}{l}830 \\
830\end{array}$ & $\begin{array}{r}11.7 \\
9.4\end{array}$ & $\begin{array}{l}109 \\
120\end{array}$ & $\begin{array}{r}92 \\
115\end{array}$ & 2.3 \\
\hline & $\begin{array}{l}\frac{\text { Control }}{1 / 8 / 60} \\
1: 45 \mathrm{pm}\end{array}$ & $\begin{array}{r}0 \\
16 \\
0\end{array}$ & $\begin{array}{r}10.5 \\
1.9 \\
12.6\end{array}$ & $\begin{array}{r}64 \\
281 \\
50\end{array}$ & $\begin{array}{l}666 \\
525 \\
635\end{array}$ & $\begin{array}{r}8.1 \\
.0 \\
10.2\end{array}$ & $\begin{array}{r}33 \\
77 \\
105\end{array}$ & $\begin{array}{r}95 \\
100 \\
105\end{array}$ & 8.1 \\
\hline & $\begin{array}{l}\text { Prog } \\
1 / 15 / 60 \\
1: 15 \mathrm{pm}\end{array}$ & $\begin{array}{r}0 \\
16 \\
8 \\
0\end{array}$ & $\begin{array}{r}14.6 \\
4.1 \\
9.6 \\
16.1\end{array}$ & $\begin{array}{r}58 \\
169 \\
78 \\
50\end{array}$ & $\begin{array}{l}842 \\
687 \\
744 \\
807\end{array}$ & $\begin{array}{r}11.6 \\
1.6 \\
6.8 \\
13.1\end{array}$ & $\begin{array}{r}104 \\
63 \\
112 \\
161\end{array}$ & $\begin{array}{l}122 \\
107 \\
110 \\
114\end{array}$ & $\begin{array}{r}10.0 \\
4.8\end{array}$ \\
\hline & $\begin{array}{l}\frac{\text { Control }}{1 / 22 / 60} \\
2: 00 \mathrm{pm}\end{array}$ & $\begin{array}{r}0 \\
16 \\
0\end{array}$ & $\begin{array}{r}11.4 \\
3.4 \\
13.2\end{array}$ & $\begin{array}{r}73 \\
211 \\
56\end{array}$ & $\begin{array}{l}833 \\
694 \\
746\end{array}$ & $\begin{array}{r}8.5 \\
0.9 \\
10.6\end{array}$ & $\begin{array}{l}89 \\
94 \\
80\end{array}$ & $\begin{array}{l}134 * \\
120 \\
143\end{array}$ & 7.6 \\
\hline & $\begin{array}{l}\frac{\text { Ald }}{1 / 29 / 60} \\
2: 45 \mathrm{pm}\end{array}$ & $\begin{array}{r}0 \\
12 \\
0\end{array}$ & $\begin{array}{r}10.4 \\
4.1 \\
10.8\end{array}$ & $\begin{array}{r}59 \\
153 \\
59\end{array}$ & $\begin{array}{l}615 \\
621 \\
634\end{array}$ & $\begin{array}{l}8.2 \\
1.9 \\
8.6\end{array}$ & $\begin{array}{l}38 \\
70 \\
79\end{array}$ & $\begin{array}{l}134 * \\
135 \\
128\end{array}$ & 6.3 \\
\hline & $\begin{array}{l}\text { Control } \\
2 / 5 / 60 \\
10: 00 \mathrm{am}\end{array}$ & $\begin{array}{r}0 \\
12 \\
8 \\
0\end{array}$ & $\begin{array}{r}15.1 \\
6.9 \\
9.2 \\
12.6\end{array}$ & $\begin{array}{r}60 \\
128 \\
89 \\
61\end{array}$ & $\begin{array}{l}908 \\
880 \\
821 \\
778\end{array}$ & $\begin{array}{r}11.9 \\
3.7 \\
6.3 \\
9.8\end{array}$ & $\begin{array}{r}75 \\
111 \\
111 \\
61\end{array}$ & $\begin{array}{l}132 \\
110 \\
106 \\
126\end{array}$ & $\begin{array}{l}8.2 \\
5.6\end{array}$ \\
\hline & $\begin{array}{l}\text { Cort } \\
\text { 4/9/60 } \\
12: 00 \mathrm{am}\end{array}$ & $\begin{array}{r}0 \\
16 \\
8 \\
0\end{array}$ & $\begin{array}{r}22.4 \\
9.9 \\
22.6 \\
26.8\end{array}$ & $\begin{array}{r}56 \\
124 \\
63 \\
47\end{array}$ & $\begin{array}{l}1265 \\
1236 \\
1418 \\
1261\end{array}$ & $\begin{array}{r}17.8 \\
5.5 \\
17.6 \\
22.4\end{array}$ & $\begin{array}{l}336 \\
215 \\
318 \\
328\end{array}$ & $\begin{array}{l}130 * \\
125 \\
132 \\
136\end{array}$ & $\begin{array}{r}16.9^{+} \\
4.8^{+}\end{array}$ \\
\hline $\begin{array}{l}\text { Mean inulin } \\
160 \mathrm{ml} \text {. pe }\end{array}$ & $\begin{array}{l}\text { earance val } \\
\text { linute after }\end{array}$ & $\begin{array}{l}\text { ues were } \\
\text { Cortisol. }\end{array}$ & $09 \mathrm{ml}$. pe & ninute for & ral & $115 \mathrm{~m}$ & per min & I Aldoste & and \\
\hline
\end{tabular}




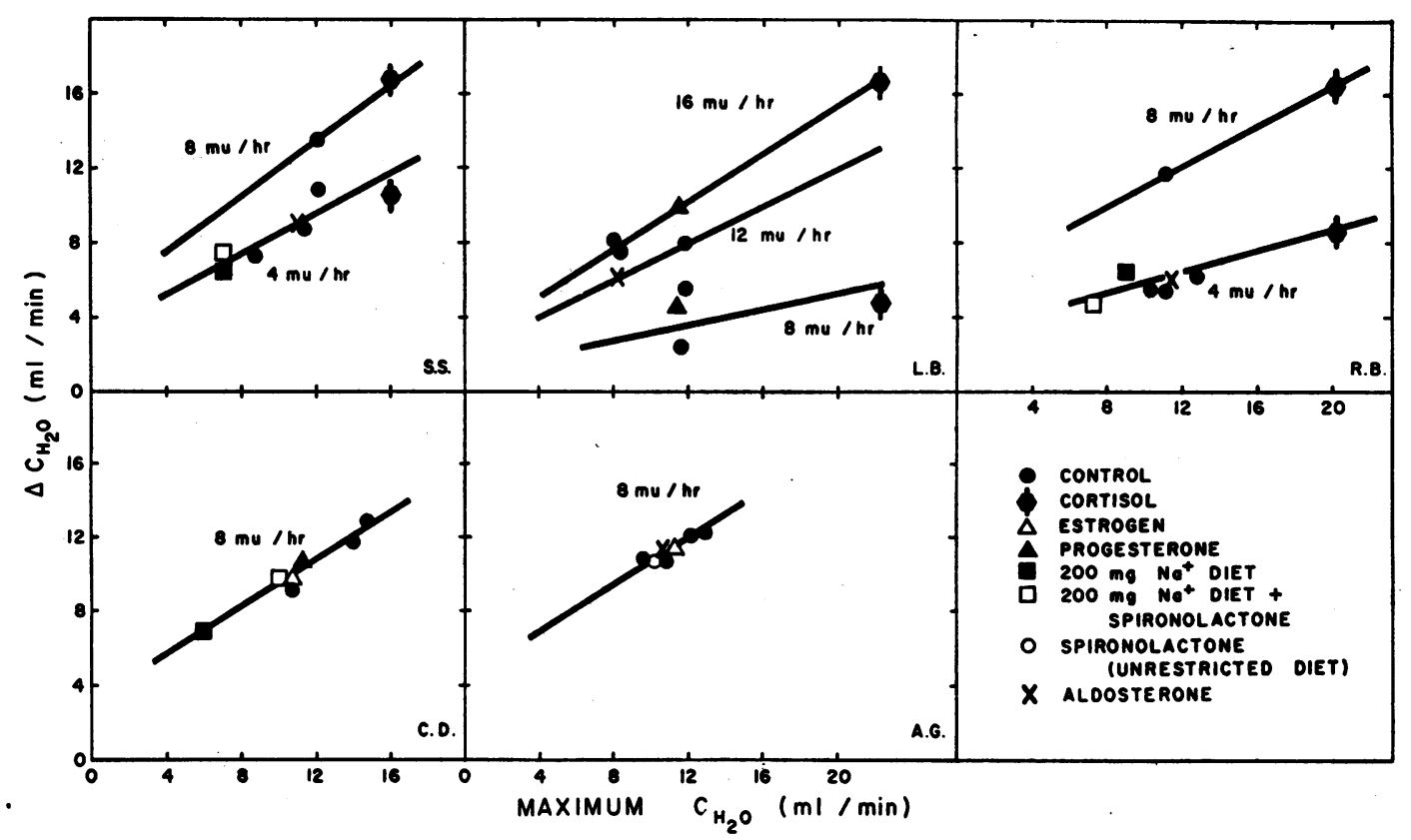

Fig. 2. Vasopressin response $\left(\Delta \mathrm{C}_{\mathrm{H}_{2} \mathrm{O}}\right.$ ) versus maximal free water clearance (maximal $\mathrm{C}_{\text {hoO }}$ ). Data are shown for subjects individually before and after the administration of cortisol, estrogen, progesterone, aldosterone, a low sodium diet and an aldosterone antagonist. The regression lines shown were drawn visually to represent the equation $\Delta \mathrm{C}_{\mathrm{H}_{2} \mathrm{O}}=k\left(\max . \mathrm{C}_{\mathrm{H}_{2} \mathrm{O}}+a\right)$. The intercept on the abscissa (value of constant $a$ ) was the same at two different dose levels in Subjects S.S., L.B. and R.B. The response to $8 \mathrm{mU}$ per hour of a less potent vasopressin preparation in L.B. was less consistent.

crease in total solute excretion and $\mathrm{C}_{\mathrm{H}_{2} \mathrm{O}}$ was observed on a low salt diet. When spironolactone was administered to these subjects, sodium excretion increased strikingly, but this was accompanied by an increase in $\mathrm{C}_{\mathrm{H}_{2} \mathrm{O}}$ and total solute excretion only in Subject C.D. In all of the foregoing experiments, the relationship between total solute excretion and $\mathrm{C}_{\mathrm{H}_{2} \mathrm{O}}$ was similar to that observed in control studies. After cortisol administration, there was a variable increase in sodium and total solute excretion; however, $\mathrm{C}_{\mathrm{H}_{2} \mathrm{O}}$ increased markedly in each subject out of proportion to the change in solute excretion.

Creatinine clearance values could not be related to changes in $\mathrm{C}_{\mathrm{H}_{2} \mathrm{O}}$ in the present study. Subject S.S. showed a rise in creatinine clearance on cortisol. In Subject L.B., inulin clearance increased on cortisol but creatinine clearance did not. Creatinine clearance was not altered consistently by any of the other treatments studied.

II. Vasopressin sensitivity. The response to a given dose of vasopressin, in terms of its effect on free water clearance $\left(\Delta \mathrm{C}_{\mathrm{H}_{2} \mathrm{O}}\right)$, was not constant in the same subject (Figure 2). As the value of $\mathrm{C}_{\mathrm{H}_{2} \mathrm{O}}$ obtained during initial maximal water diu- resis increased, the value for $\Delta \mathrm{C}_{\mathrm{H}_{2} \mathrm{O}}$ obtained in the steady state by the infusion of a given dose of vasopressin also increased. This relationship can be described by the equation:

$$
\left.\Delta \mathrm{C}_{\mathrm{H}_{2} \mathrm{O}}=k \text { (maximal } \mathrm{C}_{\mathrm{H}_{2} \mathrm{O}}+a\right)
$$

where $k$ is a constant which varies with the dose of vasopressin and $a$ is a constant amounting to 4 to $10 \mathrm{ml}$ per minute in different subjects. For the few data available, the value of $a$ appeared to be the same at different dose levels in the same subject. This relationship between $\Delta \mathrm{C}_{\mathrm{H}_{2} \mathrm{O}}$ and maximal $\mathrm{C}_{\mathrm{H}_{2} \mathrm{O}}$ accounts for much of the variability encountered both in control studies and after treatment. Thus, the increase in maximal $\mathrm{C}_{\mathrm{H}_{2} \mathrm{O}}$ after cortisol administration is accompanied by an increase in $\Delta \mathrm{C}_{\mathrm{H}_{2} \mathrm{O}}$, particularly in response to the infusion of larger doses of vasopressin.

\section{DISCUSSION}

Variations in the diuretic response to a water load which cannot be attributed to changes in endogenous antidiuretic hormone production have been described previously. These have been related to glomerular filtration rate (12), solute 
excretion $(12,13)$ and sodium excretion $(13,14)$. In the present study, a close relationship between maximal $\mathrm{C}_{\mathrm{H}_{2} \mathrm{O}}$ and total solute excretion was observed in normal subjects with diurnal, dietary and hormonally induced variations in solute excretion. It is possible that under these circumstances variations in both $\mathrm{C}_{\mathrm{H}, 0}$ and total solute excretion are determined by the amount of sodium and water delivered to the distal diluting site. Subsequent variations in reabsorption and exchange of sodium in the distal tubule could account for the lack of correlation between urinary sodium and $\mathrm{C}_{\mathrm{H}_{2} \mathrm{O}}$. In osmotic diuresis delivery of non-reabsorbable solute to the distal tubule can result in an increase in solute excretion without an associated increase in $\mathrm{C}_{\mathrm{H}_{2} \mathrm{O}}(14-16)$.

As previously noted ( 7 ), cortisol increases $\mathrm{C}_{\mathrm{H}_{2} \mathrm{O}}$ out of proportion to its effect on total solute excretion. This increase has been attributed to the effect of an increase in glomerular filtration rate, together with either a redistribution of solute reabsorption between proximal and distal tubules, or an alteration in tulbular permeability to water $(5,7)$.

A few observations are available which suggest that maximal water cliuresis is increased in pregnancy $(17,18)$. Progesterone administration has been reported to enhance the rate of water elimination in rats (19) while estrogen has no effect (6). In the present studly, progesterone and estrogen had little effect on water diuresis in normal young men. Moreover, in preliminary experiments, water diuresis showed no consistent variation at different phases of the menstrual cycle in normal young women (20).

Conceivably, aldosterone could alter the relationship between $\mathrm{C}_{\mathrm{H}, 0 \mathrm{O}}$ and solute excretion by enhancing direct sodium reabsorption at sites where free water is made available. In the present study, such an effect could not be cletected with exogenous aldosterone or when endogenous aldosterone production was increased by sodium restriction. Similarly, an aldosterone antagonist failed to decrease free water clearance. However, the variability of water diuresis in these chronic experiments may have masked slight alterations in $\mathrm{C}_{\mathrm{H}_{2} \mathrm{O}}$ caused by aldosterone.

It is possible that small changes in glomerular filtration rate could account for the changes in $\mathrm{C}_{\mathrm{H}_{2} \mathrm{O}}$ and total solute excretion observed in the present study. Creatinine clearance measurements could not detect such changes, particularly where only initial and final blood values were measured. Unfortunately, we have been unable to obtain inulin clearance measurements without interfering with maximal water diuresis in our subjects.

In the present study, $\Delta \mathrm{C}_{\mathrm{H}_{2} \mathrm{O}}$ in response to a given dose of vasopressin was found to vary with the initial value of maximal $\mathrm{C}_{\mathrm{H}_{2} \mathrm{O}}$. Hollander, Williams, Fordham and Welt (11) also noted an increase in $\Delta \mathrm{C}_{\mathrm{H}_{2} \mathrm{O}}$ in response to the same dose of vasopressin in experiments in which solute excretion was increased and initial maximal $\mathrm{C}_{\mathrm{H}_{2} \mathrm{O}}$ was high. Theoretically this relationship would be expected if the action of vasopressin were to increase the permeability of the cell membrane (21), since the amount of water reabsorbed would then depend upon the mean osmotic gradient along the distal tubule during vasopressin administration. When more free water was available in the distal tubule, the amount reabsorbed in response to vasopressin was greater, but the final urine was also more dilute. Both the lower urine osmolality and the larger value of $\Delta \mathrm{C}_{\mathrm{II}, \mathrm{O}}$ would appear to be the physicochemical consequence of the change in available free water and do not indicate a specific change in the sensitivity of the renal tubules to vasopressin. On the other hand, more endogenous anticliuretic hormone secretion may be required to concentrate the urine and maintain the water economy under conditions when maximal $\mathrm{C}_{\mathrm{H}_{2} \mathrm{O}}$ is increased. This change in requirement would apply whether $\mathrm{C}_{\mathrm{I}_{2} \mathrm{O}}$ was altered by cortisol or by any other means (15).

Whatever its theroetical basis, the observed relationship between $\Delta \mathrm{C}_{\mathrm{II}_{2} \mathrm{O}}$ and maximal $\mathrm{C}_{\mathrm{H}_{2} \mathrm{O}}$ can be used to assess variations in vasopressin response under experimental conditions. Previous studies on the effect of glucocorticoids on vasopressin response based on the effect of a single injection (7) indicated that the tubule was not insensitive to the action of $\mathrm{ADH}$. Using the more precise technique of the present study, neither cortisol nor any of the other steroids tested altered the sensitivity of the renal tubule to vasopressin.

\section{SUMMARY}

The maximal rate of water diuresis and the response to a constant infusion of vasopressin were 
studied before and after the administration of cortisol, progesterone, estrogen, aldosterone, a low sodium diet and an aldosterone antagonist in healthy young men.

Variations in free water clearance during maximal water diuresis were observed. These variations could be related to changes in total solute excretion in control experiments. This relationship was not altered by any of the treatments except cortisol administration. After cortisol administration, free water clearance increased more than would be expected from the change in solute excretion.

The decrease in free water clearance observed with the infusion of a given submaximal dose of vasopressin was directly related to the initial maximal rate of free water clearance. This relationship was unchanged by treatment, including cortisol administration.

\section{ACKNOWLEDGMENT}

We are indebted to Mrs. Kathleen Brenon, Mr. Winfred Jones and Miss Emma Rees for technical assistance and to the medical students who served as subjects.

We wish to thank C. L. Gantt, M.D., of G. D. Searle \& Co., for supplies of spironolactone (Aldactone); C. H. Sullivan, M.D., of Ciba Pharmaceutical Products, Inc., for supplies of aldosterone; and D. A. McGinty, M. D., of Parke, Davis \& Co. for reassaying our lots of Pitressin.

\section{REFERENCES}

1. Slessor, A. Studies concerning the mechanism of water retention in Addison's disease and in hypopituitarism. J. clin. Endocr. 1951, 11, 700.

2. Oleesky, S., and Stanbury, S. W. Effect of oral cortisone on water diuresis in Addison's disease and hypopituitarism. Lancet 1951, 2, 664.

3. Garrod, O., and Burston, R. A. The diuretic response to ingested water in Addison's disease and panhypopituitarism and the effect of cortisone thereon. Clin. Sci. 1952, 11, 113.

4. Garrod, O., Davies, S. A., and Cahill, G., Jr. The action of cortisone and desoxycorticosterone acetate on glomerular filtration rate and sodium and water exchange in the adrenalectomized dog. J. clin. Invest. 1955, 34, 761.

5. Kleeman, C. R., Maxwell, M. H., and Rockney, R. E. Mechanisms of impaired water excretion in adrenal and pituitary insufficiency. I. The role of altered glomerular filtration rate and solute excretion. J. clin. Invest. 1958, 37, 1799.

6. Gaunt, R., Birnie, J. H., and Eversole, W. J. Adrenal cortex and water metabolism. Physiol. Rev. 1949, 29, 281.
7. Raisz, L. G., McNeely, W. F., Saxon, L., and Rosenbaum, J. D. The effects of cortisone and hydrocortisone on water diuresis and renal function in man. J. clin. Invest. 1957, 36, 767.

8. Lloyd, C. W., and Lobotsky, J. Serum antidiuretic substances and urinary corticosteroid in the human. J. clin. Endocr. 1950, 10, 318.

9. Raisz, L. G., and Scheer, R. L. Studies on the renal concentrating mechanism. II. Effect of small acute changes in solute excretion. J. clin. Invest. 1959, $38,1$.

10. Young, M. K., Jr., and Raisz, L. G. An anthrone procedure for determination of inulin in biological fluids. Proc. Soc. exp. Biol. (N. Y.) 1952, 80, 771.

11. Hollander, W., Jr., Williams, T. F., Fordham, C. C., III, and Welt, L. G. A study of the quantitative relationship between antidiuretic hormone (vasopressin) and the renal tubular reabsorption of water. J. clin. Invest. 1957, 36, 1059.

12. Kleeman, C. R., Epstein, F. H., and White, C. The effect of variations in solute excretion and glomerular filtration on water diuresis. J. clin. Invest. 1956, 35, 749 .

13. Rosenbaum, J. D., Nelson, W. P., III, Strauss, M. B., Davis, R. K., and Rossmeisl, E. C. Variation in the diuretic response to ingested water related to the renal excretion of solutes. J. clin. Invest. 1953, 32, 394.

14. Burg, M. B., Papper, S., and Rosenbaum, J. D. Factors influencing the diuretic response to ingested water (abstract). J. clin. Invest. 1958, 37, 882.

15. Orloff, J., Wagner, H. N., Jr., and Davidson, D. G. The effect of variations in solute excretion and vasopressin dosage on the excretion of water in the dog. J. clin. Invest. 1958, 37, 458.

16. Wesson, L. G., Jr., and Anslow, W. P., Jr. Effect of osmotic and mercurial diuresis on simultaneous water diuresis. Amer. J. Physiol. 1952, 170, 255.

17. Scheer, R. L., Raisz, L. G., and Lloyd, C. W. Changes in diabetes insipidus during pregnancy and lactation. J. clin. Endocr. 1959, 19, 805.

18. Assali, N. S., Dignam, W. J., and Longo, L. Renal function in human pregnancy. III. Effects of antidiuretic hormone $(\mathrm{ADH})$ on renal hemodynamics and water and electrolyte near term and post partum. J. clin. Endocr. 1960, 20, 581.

19. Selye, H., and Bassett, L. Diuretic effect of progesterone. Proc. Soc. exp. Biol. (N.Y.) 1940, 44, 502.

20. Raisz, L. G., and Giambattista, M. A. Unpublished observations.

21. Maffly, R. H., Hays, R. M., Lamdin, E., and Leaf, A. The effect of neurohypophyseal hormones on the permeability of the toad bladder to urea. J. clin. Invest. 1960, 39, 630. 